\title{
A smartphone camera reveals an 'invisible' Parkinsonian tremor: a potential pre-motor biomarker?
}

Stefan Williams , Hui Fang, Jane Alty, Rami Qahwaji, Parisa Patel, Christopher D. Graham

Dear Sirs,

In clinical neurology, both the diagnosis and the follow-up assessment are frequently dependent upon a visual judgement made by an experienced physician. However, human vision cannot reliably measure small changes in movement. This is reflected in high false positive and negative rates for the diagnosis of disorders with prominent visual signs, such as tremor [1]].

There are a wide variety of ways to objectively detect neurological signs, but these either require special hardware (such as wearable technology) or patient behaviour change (such as engagement with smartphone tasks) [2]. Neither constraint applies to the technology of computer vision, which is the processing of single or multiple camera images by computer to automatically derive useful information. The only equipment involved is ubiquitous: camera and computer.

We report a computer vision-enhanced video sequence from a 68-year-old man, diagnosed with idiopathic Parkinson's disease 2 years previously. At the time of recording, he had Hoehn and Yahr stage 1 disease, with unilateral Parkinsonism. Examination showed mild bradykinesia and intermittent resting tremor of the left upper and lower limbs. His medication was rasagiline $1 \mathrm{mg}$ once a day, and Madopar 100/25 mg three times a day.

Eulerian magnification is a computing technique that amplifies changes in video pixels over time, so that very small motions can become visible to the human eye $[3,4]$. In ESM Fig. 1, we demonstrate how this enables an invisible tremor to become visible. The original video (ESM Fig. 1a) shows the patient's hands semipronated, in which no tremor can be seen. The amplified video (ESM Fig. 1b) is exactly the same sequence after processing with Eulerian magnification. A bilateral tremor can now be seen, which has the appearance of a Parkinsonian tremor. In contrast, for a 57-year-old healthy control participant, video amplification reveals some movement but not a Parkinsonian tremor (ESM Fig. 1c, d). 
The original videos were filmed with a smartphone camera, at 60 frames per second, under ambient light. Our computing method is a specific Eulerian video magnification algorithm, termed phase-based motion amplification [4]. In this example, we have set the amplified temporal band-passing frequency to between 3 and $7 \mathrm{~Hz}$, and the magnification factor $\alpha$ to 25 .

There are only three previous publications that apply Eulerian magnification to neurological patients $[\underline{5}, \underline{6}, \underline{7}]$. One of these features a video of the technique applied to a Parkinson's patient, but there was obvious Parkinsonism visible in the pre-processing video, and no control was shown [] $]$. Our report is the first to show Parkinsonism that is completely invisible in the original video. It demonstrates the potential to uncover subclinical neurological signs that are undetectable by the human eye alone. This raises the possibility of detecting such signs during an early, ostensibly 'pre-motor' stage of disease. We are currently working to characterise patient and control group differences after application of this algorithm.

\section{References}

1. Bajaj NPS, Gontu V, Birchall J, Patterson J, Grosset DG, Lees AJ (2010) Accuracy of clinical diagnosis in tremulous parkinsonian patients: a blinded video study. J Neurol Neurosurg Psychiatry 81:1223e1228

2. Sánchez-Ferro Á, Elshehabi M, Godinho C et al (2016) New methods for the assessment of Parkinson's disease (2005 to 2015): a systematic review. Mov Disord 31(9):1283-1292

3. Wu H, Rubinstein M, Shih E, Guttag J, Durand F, Freeman WT (2012) Eulerian video magnification for revealing subtle changes in the world. ACM Trans Graph 31(4):1-8

4. Wadhwa N, Rubinstein M, Durand F, Freeman WT (2013) Phase-based video motion processing. ACM Trans Graph 32(4):1-9.

5. Aziz NA, Tannemaat MR (2015) A microscope for subtle movements in clinical neurology. Neurology 85(10):920

6. Van Hillegondsberg L, Carr J, Brey N, Henning F (2017) Using Eulerian video magnification to enhance detection of fasciculations in people with amyotrophic lateral sclerosis. Muscle Nerve 56:1063-1067.

7. Kooij JF, van Gemert JC (2016) Depth-aware motion magnification. In: European conference on computer vision. Springer, New York, pp 467-482 